\title{
Masers as signposts of high-mass protostars
}

\section{A water maser survey of methanol maser sources ${ }^{\star}$}

\author{
M. Szymczak ${ }^{1}$, T. Pillai ${ }^{2}$, and K. M. Menten ${ }^{2}$ \\ 1 Toruń Centre for Astronomy, Nicolaus Copernicus University, Gagarina 11, 87100 Toruń, Poland \\ e-mail:msz@astro.uni.torun.pl \\ 2 Max-Planck-Institut für Radioastronomie, Auf dem Hügel 69, 53121 Bonn, Germany
}

Received 26 November 2004 / Accepted 31 December 2004

\begin{abstract}
The $22 \mathrm{GHz} \mathrm{H} \mathrm{H}_{2} \mathrm{O}$ maser line was observed towards 79 candidate high-mass protostellar objects from a flux-limited sample of $6.7 \mathrm{GHz}$ methanol sources. The emission was detected in 41 sources, towards 28 of these for the first time. The detection rate of $52 \%$ was similar to rates reported for other samples of high-mass protostars selected mainly with far-infrared (FIR) colour criteria. The median value of $\mathrm{H}_{2} \mathrm{O}$ maser luminosity of $10^{-5.5} L_{\odot}$ is equal to that of the $\mathrm{CH}_{3} \mathrm{OH}$ maser luminosity, whereas the median $\mathrm{OH}$ maser luminosity was found to be $\sim 1.5$ orders of magnitude lower. Comparison of the velocity ranges showing maser emission implies that for the majority of sources the $\mathrm{H}_{2} \mathrm{O}$ and $\mathrm{CH}_{3} \mathrm{OH}$ maser lines originate from different regions. The percentage of sources with emission in two or three of the maser species, their association with radio continuum and IR emission and the maser and IR luminosities are consistent with the view that evolutionary phases with $\mathrm{H}_{2} \mathrm{O}$ and $\mathrm{CH}_{3} \mathrm{OH}$ masers largely overlap and precede the $\mathrm{OH}$ maser phase, while at a later stage $\mathrm{OH}$ and $\mathrm{CH}_{3} \mathrm{OH}$ masers may coexist. Strong correlations of $\mathrm{OH}$ and $\mathrm{CH}_{3} \mathrm{OH}$ maser luminosities with IR luminosity and only a marginal correlation of $\mathrm{H}_{2} \mathrm{O}$ and IR luminosity confirm current pumping schemes of all three maser lines.
\end{abstract}

Key words. masers - stars: formation - ISM: molecules - radio lines: ISM - HII regions

\section{Introduction}

It has been established that the physical conditions in massive star-forming regions favour maser emission from the water $\left(\mathrm{H}_{2} \mathrm{O}\right)$, hydroxyl $(\mathrm{OH})$, and methanol $\left(\mathrm{CH}_{3} \mathrm{OH}\right)$ molecules (e.g. Kurtz et al. 2000; Reid \& Moran 1988), although the different maser species probe varying temperature, density and chemical regimes (Menten 1996, for a brief review).

The $22 \mathrm{GHz}$ transition of $\mathrm{H}_{2} \mathrm{O}$ is one of the best known and most widespread tracers of extremely young and heavily embedded low- and high-mass stars. Several single dish surveys of $\mathrm{H}_{2} \mathrm{O}$ masers were carried out towards known ultracompact HII regions (UCHIIRs) and UCHIIR candidates selected on the basis of their IRAS colours (Wouterloot \& Walmsley 1986; Churchwell et al. 1990; Palla et al. 1991, 1993; Codella et al. 1995, 1996) and also towards what Sridharan et al. (2002) and Beuther et al. (2002) identify as high-mass protostellar objects (HMPOs), i.e. sources with the same colours as UCHIIRs but with little or no radio emission. In these surveys detection rates were frequently high (up to 67\%) but strongly dependent on the sensitivity of the observations and selection criteria.

From the observations over the last decade, it has become clear that $\mathrm{H}_{2} \mathrm{O}$ maser emission marks the earliest stages of

* Table 3 is only available in electronic form at the CDS via anonymous ftp to cdsarc.u-strasbg.fr $(130.79 .128 .5)$ or via http://cdsweb.u-strasbg.fr/cgi-bin/qcat?J/A+A/434/613 high-mass star formation and, more specifically, arises in outflows from young stellar objects (Menten 1996).

High-mass star forming regions frequently display methanol maser emission. Several hundreds of methanol masers were detected towards UCHIIR candidates selected with the IRAS colour criteria of Wood \& Churchwell (1989, hereafter WC89) or other less stringent criteria (Menten 1991; Schutte et al. 1993; van der Walt et al. 1995, 1996; Walsh et al. 1997; Slysh et al. 1999; Szymczak et al. 2000) as well as in unbiased surveys of selected areas of the Galactic plane (Caswell 1996; Ellingsen et al. 1996; Szymczak et al. 2002).

These studies have shown that almost all UCHIIR candidates with extremely red IRAS colours are methanol sources, suggesting that methanol masers are closely associated with the earliest stage of high-mass star formation, in many cases with a stage preceding the formation of an UCHIIR.

Few studies have examined the relationship between $\mathrm{H}_{2} \mathrm{O}$ and $\mathrm{CH}_{3} \mathrm{OH}$ masers in the same samples of high-mass protostar candidates; see papers by Codella \& Moscadelli (2000) and Beuther et al. (2002). The detection rate of Codella \& Moscadelli (2000) was $\sim 22 \%$ for both species, but only $40 \%$ of the $\mathrm{H}_{2} \mathrm{O}$ sources had a $\mathrm{CH}_{3} \mathrm{OH}$ counterpart, indicating that at least a substantial number of sources is either in an $\mathrm{H}_{2} \mathrm{O}$ maseror a $\mathrm{CH}_{3} \mathrm{OH}$ maser bearing-phase. The formation of $\mathrm{H}_{2} \mathrm{O}$ and $\mathrm{CH}_{3} \mathrm{OH}$ masers in identical environments is precluded by very 
different excitation requirements; for $\mathrm{H}_{2} \mathrm{O}$ masers collisional pumping occurs in shocks at the interface between the outflowing material and the surrounding molecular gas (e.g. Elitzur et al. 1989), while for $\mathrm{CH}_{3} \mathrm{OH}$ masers radiative pumping (possibly) in circumstellar discs is proposed at much lower temperatures and densities (e.g. Cragg et al. 2002).

This view is confirmed by high resolution observations, which do not show spatial correlation between the two maser species in detail (on a $\sim$ sub few thousand AU scale, Forster \& Caswell 1989), although in regions harbouring masers in both species these are found within $0.15 \mathrm{pc}$ of the exciting source, if the latter is directly detected at all (Beuther et al. 2002).

In this paper we present the results of $\mathrm{H}_{2} \mathrm{O}$ maser observations of a homogeneous and unbiased sample of $6.7 \mathrm{GHz}$ $\mathrm{CH}_{3} \mathrm{OH}$ maser sources. The sample was obtained from a blind survey of the Galactic plane in the longitude range $20^{\circ}-40^{\circ}$ and latitude range from -0.52 to 0.52 (Szymczak et al. 2002). We note that the sample is flux-limited and does not suffer from other factors such as incompleteness or confusion of IR surveys and uncertainties of selection criteria, which usually affected other samples. The whole sample was recently searched for all four OH transitions at 1.6 GHz (Szymczak \& Gérard 2004).

This study continues our attempts at better characterizing the properties of the sample selected on the basis of showing 6.7 $\mathrm{GHz}$ methanol maser emission. For the reasons mentioned above, high angular resolution data are needed to study the association between $\mathrm{H}_{2} \mathrm{O}$ and $\mathrm{CH}_{3} \mathrm{OH}$ masers in individual target sources. Nevertheless, our homogeneous and large data set, although taken at low angular resolution, allows for meaningful statistical analyses of maser kinematics (via LSR velocities and velocity ranges) and luminosities. Moreover, we emphasize the "signpost effect": wherever there is a maser, there must be an exciting source. High resolution follow-up studies at radio, millimeter and infrared wavelengths will reveal these sources' properties. Our detections provide a source list for future high resolution studies.

\section{Observations and results}

\subsection{Observations}

We observed the $6_{16} \rightarrow 5_{23}(22235.0798 \mathrm{MHz})$ transition of $\mathrm{H}_{2} \mathrm{O}$ on 2003 August $12-15$ and 19 with the Effelsberg $100 \mathrm{~m}$ telescope. The half-power beam width was $40^{\prime \prime}$. During the observations the system temperature varied between 60 and $150 \mathrm{~K}$ around a median value of $92 \mathrm{~K}$. The spectrometer was split into two banks of 4096 channels, each of which was used. The bandwidth of $20 \mathrm{MHz}$ was centred on the centroid of the $\mathrm{CH}_{3} \mathrm{OH}$ velocity interval (Szymczak et al. 2002), giving an effective velocity coverage of $\sim 260 \mathrm{~km} \mathrm{~s}^{-1}$ and a spectral resolution of $0.066 \mathrm{~km} \mathrm{~s}^{-1}$. The radial velocities were measured with respect to the local standard of rest (LSR). The observations were made in the position switching mode and resulted in a mean rms noise value of $0.45 \mathrm{Jy}$ in the spectra. The calibration is estimated to be uncertain by $\sim 30 \%$.

We searched for the $\mathrm{H}_{2} \mathrm{O}$ line towards 79 targets from a sample of $\mathrm{CH}_{3} \mathrm{OH}$ masers found in the unbiased survey of the
Table 1. $22 \mathrm{GHz}$ line parameters for the new detections.

\begin{tabular}{|c|c|c|c|c|c|}
\hline \multirow[t]{2}{*}{ Source $(l, b)$} & $\Delta V$ & $V_{\mathrm{c}}$ & $V_{\mathrm{p}}$ & \multirow{2}{*}{$\begin{array}{c}S_{\mathrm{p}} \\
(\mathrm{Jy})\end{array}$} & \multirow{2}{*}{$\begin{array}{c}S_{\mathrm{i}} \\
\left(\mathrm{Jy} \mathrm{km} \mathrm{s}^{-1}\right)\end{array}$} \\
\hline & \multicolumn{3}{|c|}{$\left(\mathrm{km} \mathrm{s}^{-1}\right)$} & & \\
\hline $20.24+0.07$ & 1.1 & & 57.3 & 1.7 & 1.3 \\
\hline $21.57-0.03$ & & & 99.0 & 1.6 & 1.1 \\
\hline $22.05+0.22$ & & & 53.4 & 2.5 & 0.8 \\
\hline $23.19-0.38$ & 2.6 & 78.5 & 78.1 & 31.9 & 22.1 \\
\hline $23.26-0.24$ & 5.1 & 57.1 & 55.3 & 2.3 & 2.8 \\
\hline $23.39+0.19$ & 2.1 & & 72.0 & 3.3 & 3.5 \\
\hline $23.97-0.11$ & 42.8 & 52.4 & 32.8 & 8.8 & 14.2 \\
\hline $24.33+0.14$ & 54.5 & 85.5 & 61.7 & 7.4 & 39.6 \\
\hline $24.53+0.32$ & 7.1 & 106.0 & 103.3 & 5.5 & 6.2 \\
\hline $24.93+0.08$ & & & 47.7 & 1.1 & 1.3 \\
\hline $25.71+0.04$ & & & 63.4 & 1.2 & 0.7 \\
\hline $25.83-0.18$ & 56.4 & 94.7 & 94.9 & 338. & 754. \\
\hline $27.21+0.26$ & & & 18.6 & 4.1 & 5.9 \\
\hline $27.36-0.16$ & 16.5 & 89.5 & 95.7 & 50.6 & 121. \\
\hline $27.78+0.07$ & 33.1 & 85.2 & 69.6 & 1.1 & 2.3 \\
\hline $28.19-0.05$ & 7.4 & 92.7 & 90.7 & 7.1 & 15.6 \\
\hline $28.40+0.07$ & & & 40.1 & 2.6 & 2.6 \\
\hline $28.82+0.37$ & 7.3 & 86.8 & 87.2 & 81.5 & 93.4 \\
\hline $28.85+0.50$ & 5.5 & 87.3 & 86.9 & 12.2 & 29.4 \\
\hline $30.31+0.07$ & 4.3 & 46.6 & 47.9 & 5.6 & 9.3 \\
\hline $30.70-0.07$ & 46.7 & 76.2 & 54.7 & 1.9 & 10.8 \\
\hline $30.78+0.23$ & 7.6 & 62.9 & 39.1 & 0.5 & 1.7 \\
\hline $30.79+0.20$ & 12.4 & 70.8 & 72.0 & 5.5 & 7.7 \\
\hline $33.64-0.21$ & & & 56.4 & 1.2 & 1.5 \\
\hline $36.11+0.55$ & 10.1 & 76.7 & 72.4 & 2.5 & 5.0 \\
\hline $37.47-0.11$ & 3.8 & 61.7 & 56.7 & 3.6 & 5.5 \\
\hline $37.60+0.42$ & 11.1 & 86.3 & 90.2 & 1.1 & 5.8 \\
\hline $39.10+0.48$ & 7.2 & 27.0 & 24.4 & 1.2 & 3.5 \\
\hline
\end{tabular}

Galactic plane for the $6.7 \mathrm{GHz}$ methanol line (Szymczak et al. 2002).

\subsection{New detections}

The $22 \mathrm{GHz}$ maser line was detected in 41 sources, 28 of which are new detections. The basic parameters of the new detections and previously known masers, determined from Gaussian fitting, are summarized in Tables 1 and 2, respectively. The source designation in galactic coordinates, the velocity range of emission $(\Delta V)$, the velocity centre between the extremes $\left(V_{\mathrm{c}}\right)$, the velocity of peak emission $\left(V_{\mathrm{p}}\right)$, the flux densities of peak $\left(S_{\mathrm{p}}\right)$ and of integrated emission $\left(S_{\mathrm{i}}\right)$ are given. The same definitions hold for all types of maser observations discussed in the paper. The spectra of newly detected and previously known masers are shown in Figs. 1 and 2, respectively. Hanning smoothing was only applied to noisy spectra and reduced the resolution by a factor of 2 .

Most of the new detections are relatively weak, frequently only showing a single narrow feature. There are only three sources with a peak flux density higher than $50 \mathrm{Jy}$. Four sources have maser emission over a velocity range larger than $40 \mathrm{~km} \mathrm{~s}^{-1}$. 
Table 2. $22 \mathrm{GHz}$ line parameters for previously known masers.

\begin{tabular}{|c|c|c|c|c|c|}
\hline \multirow[t]{2}{*}{ Source $(l, b)$} & $\overline{\Delta V}$ & $V_{\mathrm{c}}$ & $V_{\mathrm{p}}$ & \multirow{2}{*}{$\begin{array}{c}S_{\mathrm{p}} \\
(\mathrm{Jy})\end{array}$} & \multirow{2}{*}{$\begin{array}{c}S_{\mathrm{i}} \\
\left(\mathrm{Jy} \mathrm{km} \mathrm{s}^{-1}\right)\end{array}$} \\
\hline & \multicolumn{3}{|c|}{$\left(\mathrm{km} \mathrm{s}^{-1}\right)$} & & \\
\hline $20.08-0.14$ & 15.6 & 24.7 & 23.6 & 18.8 & 46.4 \\
\hline $21.87+0.01$ & 4.4 & 22.3 & 23.2 & 7.0 & 12.6 \\
\hline $22.35+0.06$ & & & 88.3 & 3.6 & 3.1 \\
\hline $23.01-0.41$ & 26.0 & 79.5 & 77.0 & 206.0 & 534.6 \\
\hline $23.44-0.18$ & & & 96.4 & 11.0 & 12.2 \\
\hline $24.79+0.09$ & 94.0 & 80.0 & 112.3 & 413.0 & 1575.2 \\
\hline $27.28+0.15$ & & & 31.2 & 6.7 & 5.2 \\
\hline $29.95-0.02$ & 30.8 & 95.4 & 99.3 & 27.6 & 148.1 \\
\hline $30.59-0.04$ & 72.0 & 9.0 & 21.3 & 1.0 & 3.6 \\
\hline $30.76-0.05$ & 27.0 & 80.5 & 87.1 & 1.0 & 5.2 \\
\hline $30.82-0.05$ & 71.0 & 93.5 & 101.4 & 169.0 & 250.2 \\
\hline $33.13-0.09$ & & & 68.7 & 1.6 & 2.0 \\
\hline $37.55+0.19$ & & & 89.1 & 1.0 & 1.5 \\
\hline
\end{tabular}

\subsection{Data summary}

The properties of our 79 targets derived from the present observations and other data available in the literature are summarized in Table 3. The kinematic distances have been determined from the central velocities of the $6.7 \mathrm{GHz}$ masers as measured by Szymczak et al. (2002) and the expression for the Galactic rotation curve given by Brand \& Blitz (1993). The centre velocity, $V_{\mathrm{c}}$, of the methanol line is a reliable estimator of the systemic velocity, since it usually coincides with the velocity of CS emission (Bronfman et al. 1996), tracing dense gas, to within $4 \mathrm{~km} \mathrm{~s}^{-1}$ (Szymczak \& Gérard 2004). The sources are within the solar circle, so there are two solutions for the kinematic distances. For a few objects, unambiguous distances were taken from the literature.

The isotropic maser luminosities of the three maser molecules are derived. The $\mathrm{OH}$ maser luminosity was obtained using the sum of integrated flux densities of the 1665 and $1667 \mathrm{MHz}$ lines (Szymczak \& Gérard 2004). These main lines dominate in the studied sources, whereas the satellite lines at 1612 and $1720 \mathrm{MHz}$ are rarely seen and, if seen at all, relatively weak; therefore their contributions are ignored. The luminosity of the methanol maser emission was inferred from the integrated flux density in the $6.7 \mathrm{GHz}$ line (Szymczak et al. 2002).

The present observations are used to derive the water maser luminosity for 41 targets. Assuming that with our sensitivity we are able to detect a maser feature of velocity width of $1 \mathrm{~km} \mathrm{~s}^{-1}$ our luminosity upper limit is $2.6 \times 10^{-22} \mathrm{~W} \mathrm{~m}^{-2}$, which corresponds to $10^{-6.7} L_{\odot}$ for source at $5 \mathrm{kpc}$. $\mathrm{H}_{2} \mathrm{O}$ masers in $25.40+0.11,26.59-0.01$, and tentatively $30.82+0.27$, were recently discovered with the same instrument and similar sensitivity (Sridharan et al. 2002) and $37.53-0.11$ by Hofner \& Churchwell (1996). These four objects were not detected in the present study. Assuming "equivalent" velocity range of $1.5 \mathrm{~km} \mathrm{~s}^{-1}$ for all four objects we estimate upper limits on the ratio of the luminosities derived in the previous studies and our observations of $12.7,6.1,1.7$, and 6.8 , respectively.
Flux density variations of that order are commonly seen in water masers (Brand et al. 2003). Although detailed analysis of the variability of the $\mathrm{H}_{2} \mathrm{O}$ sources is beyond the scope of the present paper, we note similar ratios among 13 known sources in the sample. We found the highest change (increase) in the integrated flux density by a factor of 37 in 23.01-0.41 as compared to data taken at epoch 1992.99 (Valdettaro et al. 2001).

The shape of most of the spectra also exhibit substantial changes. For instance the $27.28+0.15$ maser was previously detected at $-22.5 \mathrm{~km} \mathrm{~s}^{-1}$ with a peak flux density of $\sim 0.4 \mathrm{Jy}$ (Churchwell et al. 1990) while we detected only a new feature of a $5.9 \mathrm{Jy}$ peak flux density at $31.2 \mathrm{~km} \mathrm{~s}^{-1}$.

To determine the IR luminosity, we identified candidate IRAS source counterparts to within $1^{\prime}$ from the methanol position (Szymczak et al. 2002). We then estimated the total IR flux using the relation given by Walsh et al. (1997) (their Eq. (3)) adopting the same factor $Y=0.6$ to incorporate the flux from outside the IRAS wavelength range.

Radio continuum counterparts of our targets were identified within $1^{\prime}$ from the methanol position using mainly the VLA $5 \mathrm{GHz}$ catalogue of UCHIIRs (Becker et al. 1994). Sources with radio continuum emission are marked in the last column of Table 3.

\section{Discussion}

\subsection{Detection statistics}

$\mathrm{H}_{2} \mathrm{O}$ masers were detected in 52\% (41/79) of the sources in our $\mathrm{CH}_{3} \mathrm{OH}$ sample (Table 3). Sridharan et al. (2002) reported a detection rate of $42 \%$ for their sample of 69 high-mass protostellar objects, which are basically bright IRAS point sources with colours typical of UCHIIRs with detected CS emission and without significant radio emission at $5 \mathrm{GHz}$. They carried out the observations with the same instrument and sensitivity as we. Our sample has only three sources in common with that of Sridharan et al. It appears that the detection rates for both samples do not differ significantly. However, the real difference in the detection rates might be larger and our value should be regarded as a lower limit, since the errors on our methanol positions of about $30^{\prime \prime}$ for strong sources and as large as 70" for weak sources (Szymczak et al. 2002) are comparable to or larger than the $40^{\prime \prime}$ beamwidth of our $\mathrm{H}_{2} \mathrm{O}$ observation: we simply may have missed sources. As we did not make raster mapping to find peak position our integrated flux density estimates may by quite uncertain as well (see Sect. 3.3).

Sridharan et al. used the IRAS positions, and consequently their data do not suffer as much from that effect, although many IRAS positions have uncertainties $>10^{\prime \prime}$. In our sample we note a considerable decrease of the $\mathrm{H}_{2} \mathrm{O}$ maser detection rate for the sources with weak methanol emission. This can be due to inaccurate positions of methanol sources. However, it could be also related to a correlation of luminosities of both maser lines (see Sect. 3.3). Finally, variability may play a role, which is difficult to quantify.

Churchwell et al. (1990) detected $\mathrm{H}_{2} \mathrm{O}$ masers in $67 \%$ of 84 IRAS colour-selected UCHIIRs showing radio continuum emission. The sensitivity of their observations was about 

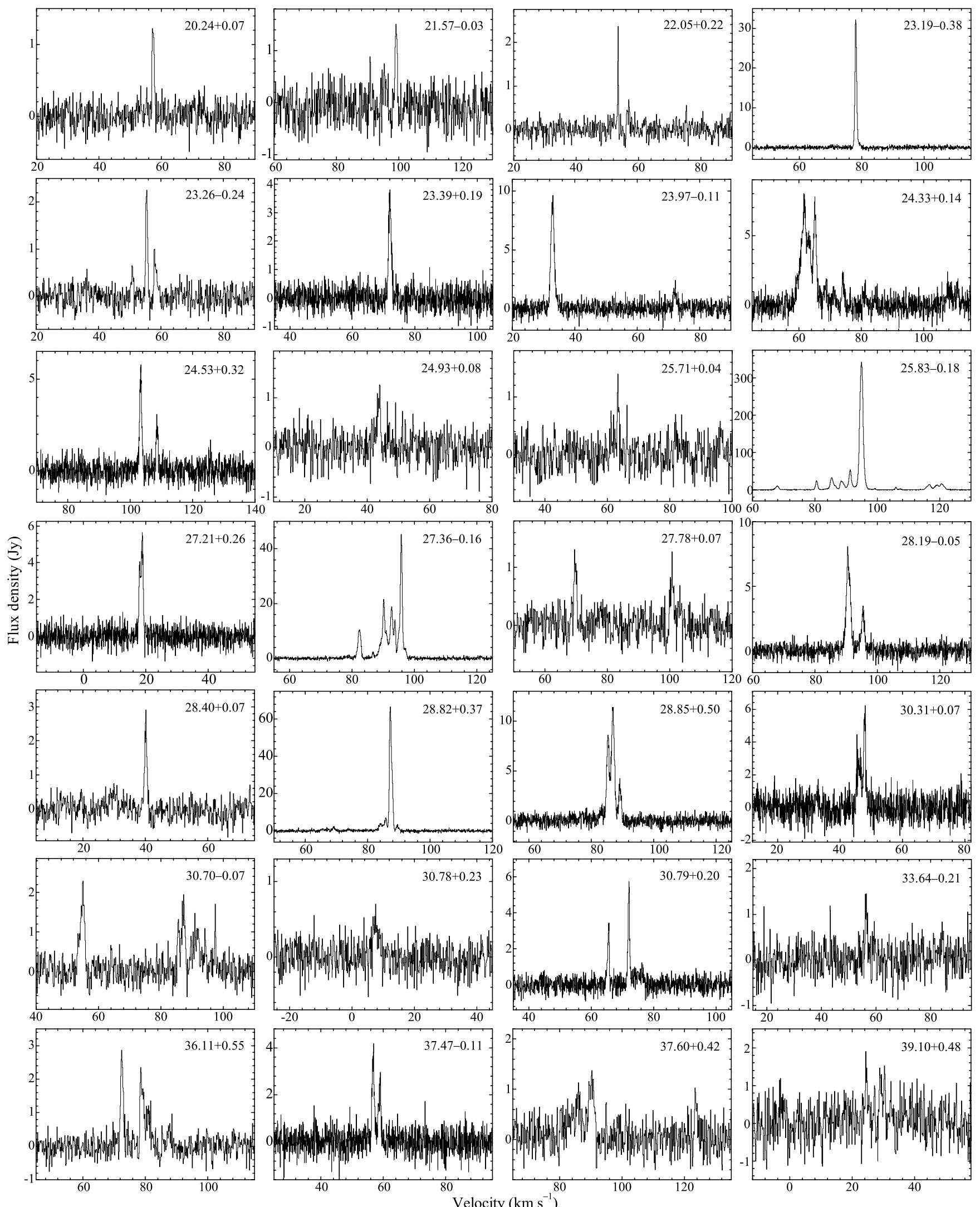

Fig. 1. Spectra of the newly detected water masers. 

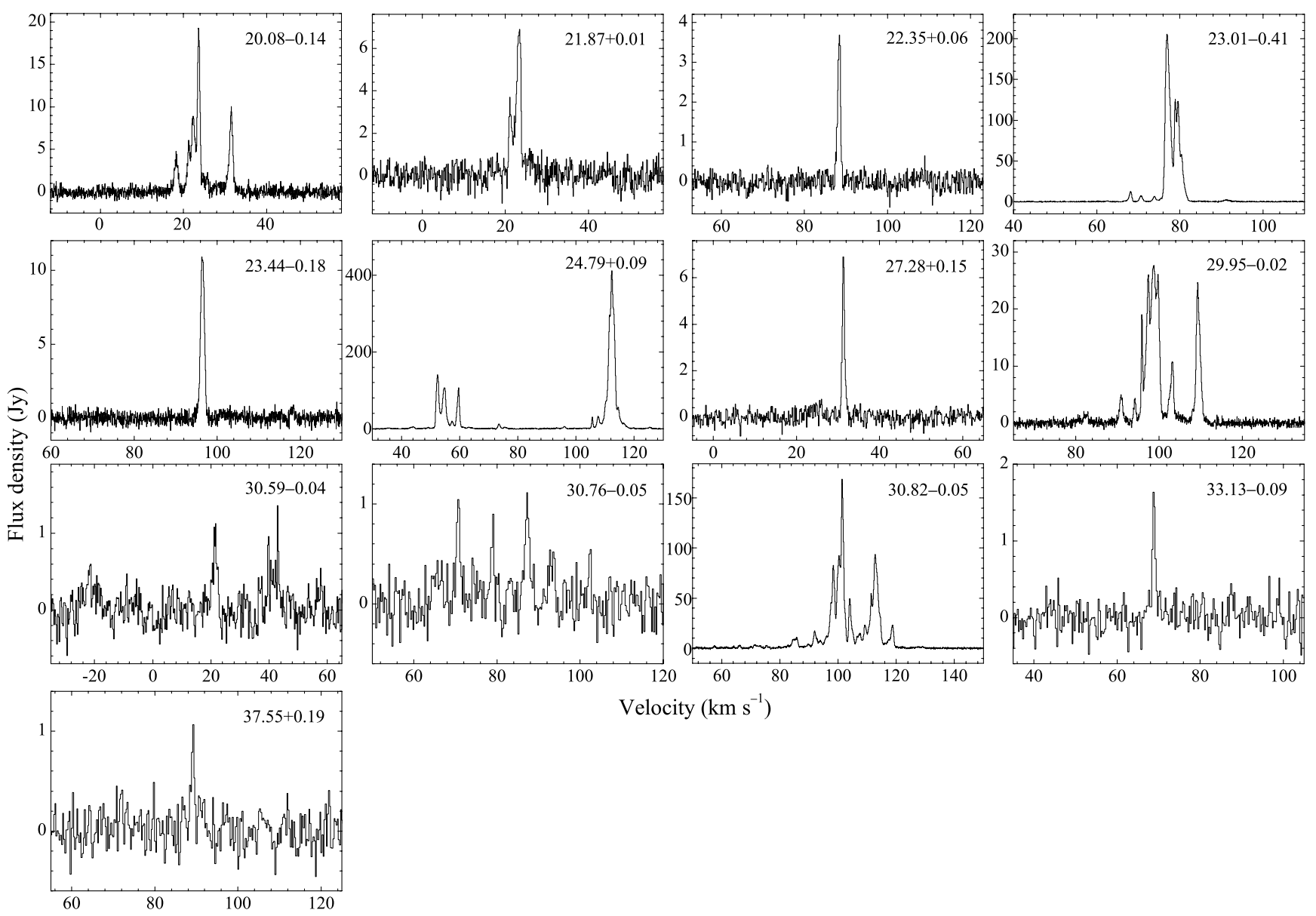

Fig. 2. Spectra of previously known water masers.

a factor of 2.7 better than ours. When scaled to the sensitivity of our observations their detection rate is reduced to 53\%, which is in excellent agreement with the present detection rate. We note that Churchwell et al.'s sample is largely independent from ours, with only 7 objects in common. Therefore, it is very likely that both samples probe the same population of objects with a high probability of the occurrence of $\mathrm{H}_{2} \mathrm{O}$ maser emission. Palla et al. (1991) investigated the $\mathrm{H}_{2} \mathrm{O}$ maser properties of a sample of 260 bright IRAS objects. For about half of their sources (125), which satisfied the WC89 colour criteria, the detection rate was $26 \%$. We note that in our survey there is a large number of maser sources well below their detection limit $(3 \sigma)$ of about $5 \mathrm{Jy}$.

When our data are scaled to their sensitivity the detection rate would decrease to $25 \%$. Thus for both samples the percentage of $\mathrm{H}_{2} \mathrm{O}$ masers is nearly the same. We conclude that the percentage of $\mathrm{H}_{2} \mathrm{O}$ masers in the unbiased sample of methanol sources is nearly the same as that reported for the above three largely independent samples of high-mass protostar candidates selected by different criteria. The $6.7 \mathrm{GHz}$ methanol maser line is as powerful a tool to probe high-mass star forming regions and identify high-mass protostars as methods based on far-infrared, radio continuum, and thermal line data (WC89; Sridharan et al. 2002).

Water maser emission generally exhibits strong flux variations (e.g. Brand et al. 2003), which can influence the detection rate. It is very likely that the four known sources mentioned in Sect. 2.3 were not detected due to variability. This implies that the detection rate can vary by $5-6 \%$.

We suspect that the number of $\mathrm{H}_{2} \mathrm{O}$ masers in the sample detected at least at some epochs could be increased if not detected sources were reobserved.

\subsubsection{Sources with masers in different species}

With the completion of the present survey we possess data for the three major maser species for a medium-size sample of objects. This allows us to deduce information on the occurrence or non-occurrence of maser emission in different species.

To do this we applied a common sensitivity of $1.6 \mathrm{Jy}$, which is a cut-off level for the unbiased $\mathrm{CH}_{3} \mathrm{OH}$ survey (Szymczak et al. 2002), for OH data (Szymczak \& Gérard 2004) and the present $\mathrm{H}_{2} \mathrm{O}$ observations. Table 4 summarizes the number and percentage of sources with masers from more then one species together with radio and IRAS properties.

The percentage of sources with both methanol and water masers (44\%) in our sample is larger than the $29 \%$ in Sridharan et al.'s (2002) sample. However, for their subsample of 29 objects observed with a few arcsecond resolution this percentage is 56\% (Beuther et al. 2002). Codella \& Moscadelli (2000) found that about $40 \%$ of $\mathrm{H}_{2} \mathrm{O}$ masers associated with UCHIIRs 
Table 4. Statistics on the overlap of maser species, radio continuum emission and IRAS emission. The third column gives the percentage of sources with masers in two or three of the maser species $(\mathrm{OH}$, $\mathrm{H}_{2} \mathrm{O}$, and $\mathrm{CH}_{3} \mathrm{OH}$ ) with respect to the total number of sources studied (79). The fifth and seventh columns are the percentages of radio and IRAS counterparts, respectively, with respect to the number of maser sources given in the second column.

\begin{tabular}{lcccccc}
\hline \hline Overlap & $N_{\text {maser }}$ & $\%$ & $N_{\text {radio }}$ & $\%$ & $N_{\text {IRAS }}$ & $\%$ \\
\hline $\mathrm{OH} / \mathrm{CH}_{3} \mathrm{OH} / \mathrm{H}_{2} \mathrm{O}$ & 9 & 11 & 4 & 44 & 5 & 56 \\
$\mathrm{OH} / \mathrm{CH}_{3} \mathrm{OH}$ & 12 & 15 & 4 & 33 & 4 & 33 \\
$\mathrm{CH}_{3} \mathrm{OH} / \mathrm{H}_{2} \mathrm{O}$ & 26 & 33 & 10 & 38 & 14 & 54 \\
$\mathrm{CH}_{3} \mathrm{OH}$ & 32 & 41 & 6 & 19 & 18 & 56 \\
\hline
\end{tabular}

with detected radio continuum emission have $\mathrm{CH}_{3} \mathrm{OH}$ counterparts.

Although association of both maser species in the sample might be partly caused by insufficient angular resolution we notice a high fraction of sources showing both $\mathrm{CH}_{3} \mathrm{OH}$ and $\mathrm{H}_{2} \mathrm{O}$ emission. As this fraction is comparable to that reported for the above mentioned samples we suggest that $\mathrm{CH}_{3} \mathrm{OH}$ maser efficiently probes the same population of high mass star-forming regions as do far infrared colour criteria (WC89). In the same population, evolutionary phases in which, both, $\mathrm{CH}_{3} \mathrm{OH}$ and $\mathrm{H}_{2} \mathrm{O}$ emission is produced, can overlap significantly (Codella \& Moscadelli 2000) and both masers likely originate in similar environments, although the phenomena actually producing the maser emission may be quite different - warm, moderately dense gas for $\mathrm{CH}_{3} \mathrm{OH}$ and hot, shocked, dense gas for $\mathrm{H}_{2} \mathrm{O}$ (Menten 1996). Masers from different species may arise from different young stellar objects within the same protostellar cluster. The correlations discussed in the following sections should be interpreted only as presence of a given maser type in the star-forming complex during its evolution.

Forster \& Caswell (1989) established that $\mathrm{OH}$ and $\mathrm{H}_{2} \mathrm{O}$ masers closely associate in compact groups of size of $\sim 0.03 \mathrm{pc}$, whereas in more extended groups $(\sim 0.15 \mathrm{pc})$ are spatially intermixed but usually do not coincide. They propose a scenario that close $\mathrm{OH} / \mathrm{H}_{2} \mathrm{O}$ associations last about $10^{5} \mathrm{yr}$ during an accretion phase. When accretion terminates $\mathrm{OH} / \mathrm{H}_{2} \mathrm{O}$ groups expand over the next $10^{4} \mathrm{yr}$ and then disappear. Caswell et al. (1995) showed that $\mathrm{OH}$ and $\mathrm{CH}_{3} \mathrm{OH}$ masers are very closely associated and generally occur near a common energy source.

A view of the $\mathrm{W} 3(\mathrm{OH})$ region (Menten 1996) provides lessons applicable here. $\mathrm{OH}$ and $\mathrm{CH}_{3} \mathrm{OH}$, but no $\mathrm{H}_{2} \mathrm{O}$ maser emission is observed towards half of the ultracompact (size $\approx$ $2000 \mathrm{AU}$ ) HII region radio emission. In contrast, very intense $\mathrm{H}_{2} \mathrm{O}$ maser emission is observed towards a separate protostar or cluster of protostars $0.06 \mathrm{pc}$ (or 6") away, which also gives rise to strong thermal molecular and dust emission (Wyrowski et al. 1999). At the resolution of the IRAS satellite these sources cannot be separated. Needless to say, with our coarse resolution, in particular at $6.7 \mathrm{GHz}, \mathrm{H}_{2} \mathrm{O}$ and $\mathrm{CH}_{3} \mathrm{OH}$ masers would appear as coextensive.
The probability of finding an $\mathrm{OH}$ maser counterpart of a $\mathrm{CH}_{3} \mathrm{OH} / \mathrm{H}_{2} \mathrm{O}$ maser source is relatively low (Table 4). This may suggest less overlap of their evolutionary phases as compared to overlap of the $\mathrm{CH}_{3} \mathrm{OH}$ and $\mathrm{H}_{2} \mathrm{O}$ maser phases. Further arguments supporting this suggestion are presented in Sect. 3.3.

\subsubsection{Association with radio and IRAS sources}

Out of 79 sources, 24 (30\%) are associated with radio continuum emission (Tables 3 and 4). This percentage may be an overestimate, again, due to the relatively high uncertainty of the methanol positions. From high resolution data, Walsh et al. (1998) derive that only $20 \%$ of methanol masers are accompanied by radio continuum. Beuther et al. (2002) found continuum emission at centimeter wavelengths in about $31 \%$ of sources with $\mathrm{CH}_{3} \mathrm{OH}$ and $\mathrm{H}_{2} \mathrm{O}$ masers. The presence of radio continuum is thought to be a signpost of high-mass stars that have recently ignited and should, thus, indicate more evolved objects. Therefore, a prevalence of $\mathrm{CH}_{3} \mathrm{OH}$ and $\mathrm{H}_{2} \mathrm{O}$ masers without detectable radio continuum emission, in the three almost independent samples of high mass protostar candidates, implies that most of them are in the earliest stages of evolution.

Alternatively, some of the objects may be associated with less massive stars where the ionization rates are too low to produce observable UCHIIRs. The latter possibility is less probable as generally no methanol emission was detected towards low-mass young stellar objects (Minier et al. 2003), whereas such objects frequently show very intense $\mathrm{H}_{2} \mathrm{O}$ maser emission (e.g. Valdettaro et al. 2001). Objects with only $\mathrm{CH}_{3} \mathrm{OH}$ maser emission (Table 4) have the lowest percentage of radio counterparts (19\%). This seems to be consistent with Beuther et al.'s (2002) result and may suggest that due to the ignition of the central object the $\mathrm{CH}_{3} \mathrm{OH}$ maser emission ceases before the $\mathrm{H}_{2} \mathrm{O}$ maser emission. However, there are well-known counter examples, perhaps most prominently $\mathrm{W} 3(\mathrm{OH})$ discussed above.

There are only two sources $24.51-0.05$ and $28.31-0.40$ with only $\mathrm{OH}$ maser and radio emission. We cannot state, however, that they are more evolved than other our targets. More than $50 \%$ of the sources are associated with an IRAS point source (Table 4).

This is consistent with results reported by Ellingsen et al. (1996) and Beuther et al. (2002). The percentage of methanol sources associated with mid-infrared emission (MIR) is $\sim 70 \%$ (Walsh et al. 2001) as IRAS point sources do not always coincide with the MIR sources possibly due to significant confusion.

\subsection{Velocities}

The distributions of velocity ranges of $\mathrm{OH}$ and $\mathrm{CH}_{3} \mathrm{OH}$ masers in the sample do not differ (Fig. 3); the averages are $8.0 \pm 0.7$ and $8.6 \pm 0.5 \mathrm{~km} \mathrm{~s}^{-1}$, respectively. $\Delta V$ of these masers is never larger than $20 \mathrm{~km} \mathrm{~s}^{-1}$. In contrast, out of $45 \mathrm{H}_{2} \mathrm{O}$ sources $33 \%$ have $\Delta V$ larger than $20 \mathrm{~km} \mathrm{~s}^{-1}$ while in $22 \%$ of sources its value exceeds $40 \mathrm{~km} \mathrm{~s}^{-1}$. This suggests that at least one third of the water masers in the sample originate from environments different than the methanol masers and some of them can be associated with outflows or jets. 


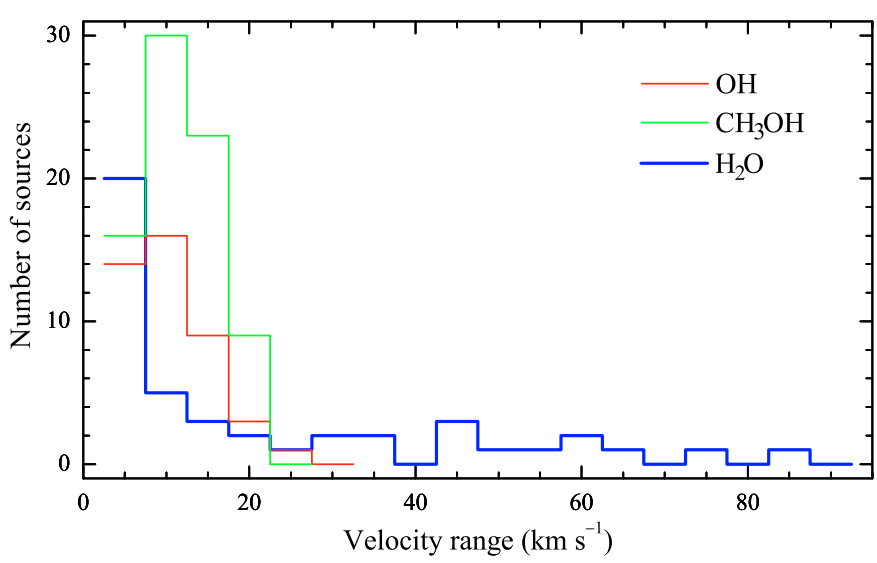

Fig. 3. Histograms of the velocity extent of three maser lines.

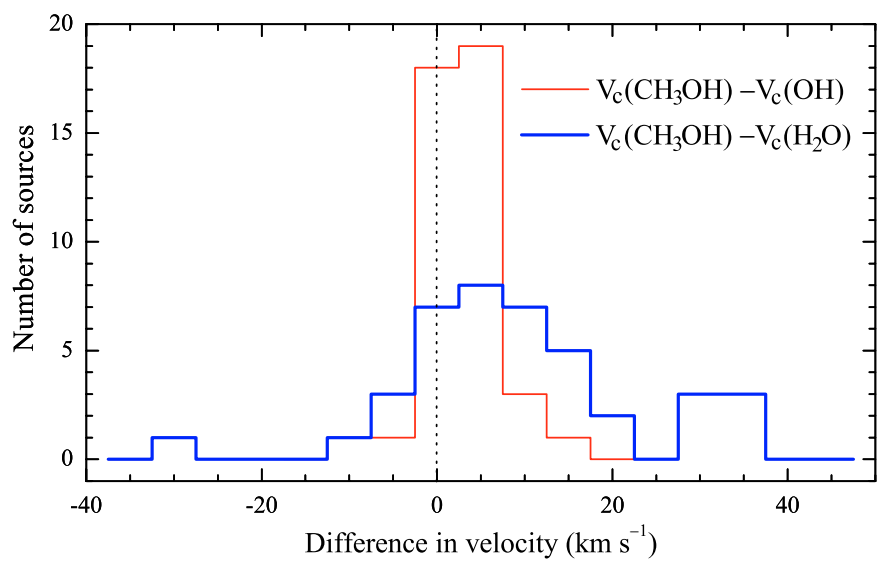

Fig. 4. Histograms of the difference in the centroid of the velocity maser intervals.

The distributions of the difference in $V_{\mathrm{c}}$ between $\mathrm{CH}_{3} \mathrm{OH}$ and $\mathrm{OH}$ and between $\mathrm{CH}_{3} \mathrm{OH}$ and $\mathrm{H}_{2} \mathrm{O}$ are shown in Fig. 4. Out of $43 \mathrm{OH}$ masers, $88 \%$ have velocity differences smaller than $5 \mathrm{~km} \mathrm{~s}^{-1}$. Among $\mathrm{H}_{2} \mathrm{O}$ masers, about $61 \%$ have velocity differences less than $10 \mathrm{~km} \mathrm{~s}^{-1}$ and $37 \%$ have differences less than $5 \mathrm{~km} \mathrm{~s}^{-1}$. The small velocity difference between $\mathrm{CH}_{3} \mathrm{OH}$ and $\mathrm{OH}$ implies that the maser lines of both molecules emerge either from the same regions of material surrounding the central object or from different clumps which are not strongly perturbed by the neutral shocks. This interpretation is consistent with high angular resolution observations of $\mathrm{W} 3(\mathrm{OH})$ (Menten et al. 1992), where the distribution of the $6.7 \mathrm{GHz}$ methanol masers follows closely that of the $1665 \mathrm{MHz} \mathrm{OH}$ masers.

Figure 4 implies that the majority of the $\mathrm{H}_{2} \mathrm{O}$ masers originate in regions of velocities considerably different from the methanol velocities. As already discussed above, high resolution data indicate that there are clear spatial separations between both maser types (Beuther et al. 2002). Moreover, the velocity differences can reflect different excitation conditions. The excitation of $\mathrm{H}_{2} \mathrm{O}$ maser is due to collisions with $\mathrm{H}_{2}$ molecules within shocks associated with outflows or accretion (e.g. Elitzur et al. 1989) and requires densities, $n$, of $\sim 10^{9} \mathrm{~cm}^{-3}$ and temperatures, $T$, of $\sim 400 \mathrm{~K}$.

Radiative pumping in dense circumstellar gas (e.g. discs) is favoured for $\mathrm{CH}_{3} \mathrm{OH}$ masers (e.g. Cragg et al. 2002, find

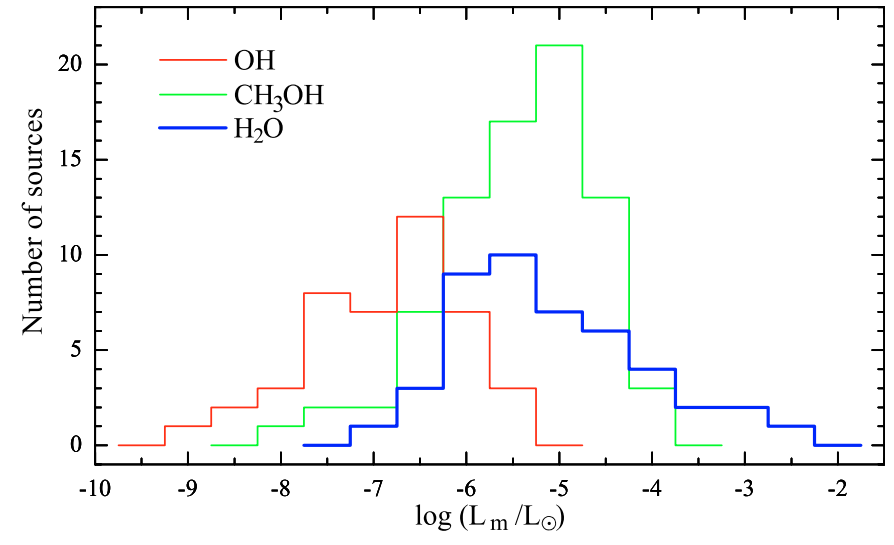

Fig. 5. Histograms of the maser luminosity for three species assuming the near kinematic distance.

$n=10^{5}-10^{8} \mathrm{~cm}^{-3}$ and $\left.T \approx 100 \mathrm{~K}\right)$. The distributions in Fig. 4 are shifted to positive values; the centroids of the $\mathrm{OH}$ and $\mathrm{H}_{2} \mathrm{O}$ maser velocity intervals are generally blue-shifted relative to the centroids of the methanol velocity intervals being good estimates of the systemic velocities if one assumes that $\mathrm{CH}_{3} \mathrm{OH}$ masers originate in discs. If all three lines are associated with the same central object, this shift implies that $\mathrm{OH}$ and $\mathrm{H}_{2} \mathrm{O}$ masers are seen preferentially in front of the central object. $\mathrm{CH}_{3} \mathrm{OH}$ masers are likely confined to the innermost dense parts of the environment of a high-mass star, while $\mathrm{OH}$ originates in less dense outer clouds and $\mathrm{H}_{2} \mathrm{O}$ marks the high velocity part of outflows (Codella et al. 2004). The prevalence of the blue-shifted methanol maser emission may indicate that it arises from infalling gas.

\subsection{Luminosities}

Although the near-far kinematic distance ambiguity was largely not resolved, near kinematic distance are more likely for a large number of the sources listed in Table 3 (Churchwell et al. 1990; Walsh et al. 1997). The luminosity distributions for the three maser species shown in Fig. 5 were derived assuming the near kinematic distances. The median luminosity of $\mathrm{H}_{2} \mathrm{O}$ masers is $10^{-5.5} L_{\odot}$ and equals that of $\mathrm{CH}_{3} \mathrm{OH}$ masers.

The $\mathrm{H}_{2} \mathrm{O}$ luminosity distribution is clearly skewed to larger values, whereas that of the $\mathrm{CH}_{3} \mathrm{OH}$ masers is skewed to smaller values, resulting in average luminosities of $10^{-5.3}$ and $10^{-5.7} L_{\odot}$, respectively. There is no statistically significant difference between the luminosities of $\mathrm{H}_{2} \mathrm{O}$ and $\mathrm{CH}_{3} \mathrm{OH}$ masers. The average $\mathrm{OH}$ luminosity is $10^{-7.1} L_{\odot}$. We state that while the luminosities of $\mathrm{H}_{2} \mathrm{O}$ and $\mathrm{CH}_{3} \mathrm{OH}$ masers are comparable, $\mathrm{OH}$ masers are less luminous by about 1.5 orders of magnitude. Because we did not make raster maps to find peak positions, our estimate of $\mathrm{H}_{2} \mathrm{O}$ luminosity may be underestimated. In order to estimate this effect we compared our flux densities of 13 previously known sources with those published in the literature. The mean of ratios of our measurements and other authors' is $8.2 \pm 16.0$.

Distributions of the $\mathrm{H}_{2} \mathrm{O}$ maser luminosity for the present sample and that of Sridharan et al. (2002) are shown in Fig. 6. No significant difference is seen between the samples. 
Table 5. The average maser and IR luminosities.

\begin{tabular}{lcccccc}
\hline \hline Overlap & $N_{\text {maser }}$ & $\log \left(L_{\mathrm{OH}}\right)$ & $\log \left(L_{\mathrm{CH}_{3} \mathrm{OH}}\right)$ & $\log \left(L_{\mathrm{H}_{2} \mathrm{O}}\right)$ & $N_{\mathrm{IR}}$ & $\log \left(L_{\mathrm{IR}}\right)$ \\
\hline $\mathrm{OH} / \mathrm{CH}_{3} \mathrm{OH} / \mathrm{H}_{2} \mathrm{O}$ & 9 & $-6.32 \pm 0.14$ & $-5.44 \pm 0.32$ & $-4.72 \pm 0.44$ & 5 & $4.62 \pm 0.20$ \\
$\mathrm{OH} / \mathrm{CH}_{3} \mathrm{OH}$ & 12 & $-6.72 \pm 0.22$ & $-5.29 \pm 0.24$ & & 4 & $5.12 \pm 0.25$ \\
$\mathrm{CH}_{3} \mathrm{OH} / \mathrm{H}_{2} \mathrm{O}$ & 26 & & $-5.60 \pm 0.15$ & $-5.32 \pm 0.19$ & 14 & $4.61 \pm 0.19$ \\
$\mathrm{CH}_{3} \mathrm{OH}$ & 32 & & $-5.94 \pm 0.13$ & & 18 & $3.76 \pm 0.19$ \\
\hline
\end{tabular}

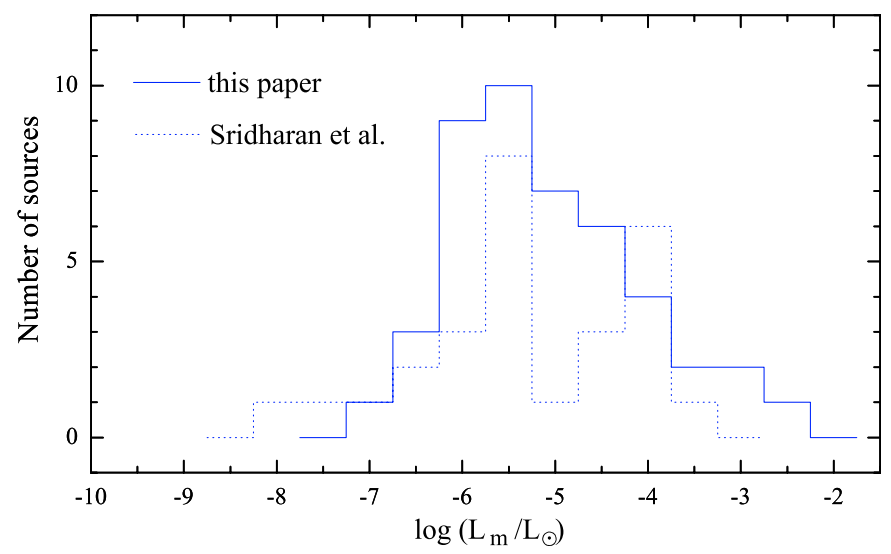

Fig. 6. Histograms of the $\mathrm{H}_{2} \mathrm{O}$ maser luminosity for our sample and that of Sridharan et al. (2002).

The median value of $L_{\mathrm{H}_{2} \mathrm{O}}$ of Sridharan et al.'s sample is $10^{-5.6} L_{\odot}$, which is essentially the same as our value. This suggests that the two samples probe similar population of young stellar objects and that our observations did not seriously underestimate the $\mathrm{H}_{2} \mathrm{O}$ luminosities. However, more precise observations are needed to confirm this.

For the subsets of sources described in Sect. 3.1.1 (Table 4) the average maser luminosities are given in Table 5. We found for the subset of $\mathrm{OH} / \mathrm{CH}_{3} \mathrm{OH} / \mathrm{H}_{2} \mathrm{O}$ sources the $\mathrm{OH}$ luminosity to be statistically significantly lower than the $\mathrm{CH}_{3} \mathrm{OH}$ $(p<0.02)$ and $\mathrm{H}_{2} \mathrm{O}(p<0.003)$ luminosities, respectively. A strong difference in maser luminosities is also seen in the subset of $\mathrm{OH} / \mathrm{CH}_{3} \mathrm{OH}$ objects $(p<0.0003)$. The $\mathrm{CH}_{3} \mathrm{OH}$ luminosity significantly differs between the $\mathrm{OH} / \mathrm{CH}_{3} \mathrm{OH}$ and $\mathrm{CH}_{3} \mathrm{OH}$ maser groups. We conclude that the $\mathrm{H}_{2} \mathrm{O}$ and $\mathrm{CH}_{3} \mathrm{OH}$ maser luminosities are comparable within the error ranges, while the $\mathrm{OH}$ luminosity is always lower than the luminosities of the two other maser species. Models predict that $\mathrm{OH}$ and $\mathrm{CH}_{3} \mathrm{OH}$ masers operate simultaneously under a wide range of physical conditions (Cragg et al. 2002). Our findings, on the other hand, suggest that $\mathrm{OH}$ maser emission appears in evolutionary phase later than $\mathrm{CH}_{3} \mathrm{OH}$ and $\mathrm{H}_{2} \mathrm{O}$ maser emission.

This is consistent with predictions of chemical models (Charnley et al. 1995; Charnley 1997). On the other hand if the luminosity ratio of $\mathrm{OH}$ and $\mathrm{CH}_{3} \mathrm{OH}$ masers is constant then one would detect very weak $\mathrm{OH}$ masers associated with the subset of sources with $\mathrm{CH}_{3} \mathrm{OH}$ masers of low luminosity. Such weak $\mathrm{OH}$ masers would be below the detection limit of the $\mathrm{OH}$ data available to us.

The subset of sources with $\mathrm{CH}_{3} \mathrm{OH}$ alone has a significantly lower IR luminosity than that of the other subsets. The same trend is observed for the maser luminosity. As shown in

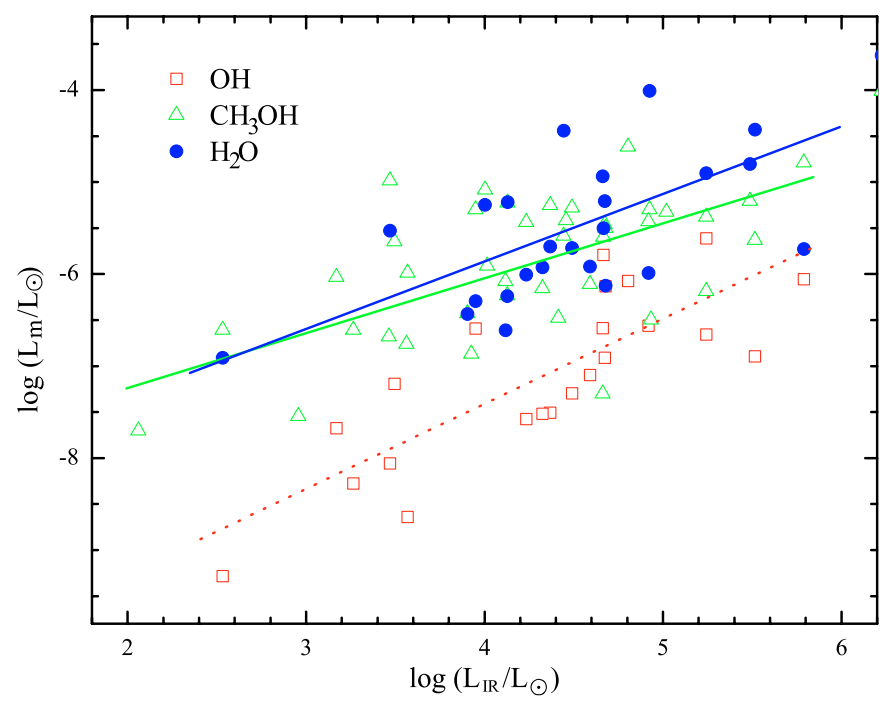

Fig. 7. Maser luminosities versus infrared luminosity. The least squares fits to the data are shown by dashed (red), thin (green) and thick (blue) lines for $\mathrm{OH}, \mathrm{CH}_{3} \mathrm{OH}$ and $\mathrm{H}_{2} \mathrm{O}$ sources, respectively.

Sect. 3.1.2 this group of objects is rarely associated with detectable radio continuum emission. We suggest that a significant fraction of the sources with only methanol maser emission, constituting more than $40 \%$ of the sample, precede in evolution those high-mass star forming sites associated with $\mathrm{OH}$ and UCHIIRs and some of them may be at very early stages.

Figure 7 shows the luminosities of three maser species versus the IR luminosity. The strongest correlation is seen for $\mathrm{OH}$ masers $(r=0.84, p<0.0001)$. $\mathrm{CH}_{3} \mathrm{OH}$ luminosity is also correlated with IR luminosity $(r=0.55, p<0.0001)$. However, $\mathrm{H}_{2} \mathrm{O}$ and IR luminosities are only marginally correlated $(r=0.50, p<0.04)$. A tight dependence of $\mathrm{OH}$ and $\mathrm{CH}_{3} \mathrm{OH}$ luminosities on IR luminosity does not necessarely imply that IR photons are involved in their pumping. It reflects rather the fact that more luminous star forming complexes provide more energy for the maser pumping. A correlation of $\mathrm{H}_{2} \mathrm{O}$ luminosity with IR widely reported in the past (e.g. Wouterloot \& Walmsley 1986; Palla et al. 1991) is not confirmed in our study. This may imply that IR photons do not influence $\mathrm{H}_{2} \mathrm{O}$ maser pumping, as was suggested previously (Wouterloot \& Walmsley 1986), which also agrees with feasible pumping schemes (Elitzur et al. 1989).

Analysis of the IRAS colour-colour diagram of the sample does not provide any new information over that reported in Szymczak et al. (2002) and Beuther et al. (2002). Sources having different maser combinations are clustered in the reddest part of diagram corresponding to less evolved objects. 


\section{Conclusions}

A high sensitivity survey of the $\mathrm{H}_{2} \mathrm{O}$ maser line in an unbiased sample of 79 methanol maser sources resulted in 41 detections of which 28 are new. The water maser detection rate was $52 \%$, which is comparable with the rates reported for samples of high-mass star-forming regions selected with other criteria mainly based on IRAS colours typical for UCHIIRs or radio continuum emission or/and thermal CS emission.

The sample was divided into four subsets, depending whether maser lines from $\mathrm{OH}, \mathrm{CH}_{3} \mathrm{OH}$, and $\mathrm{H}_{2} \mathrm{O}$ were observed in a source. Percentages of sources masing in two or three of the species or one only, as well as an association of maser and radio continuum and/or IR emission were analyzed, as well as the maser and IR luminosities.

We find that $\mathrm{CH}_{3} \mathrm{OH}$ and $\mathrm{H}_{2} \mathrm{O}$ evolutionary phases largely overlap and luminosities in both species are comparable. Only a small fraction $(\sim 10 \%)$ of $\mathrm{CH}_{3} \mathrm{OH} / \mathrm{H}_{2} \mathrm{O}$ objects have $\mathrm{OH}$ emission detectable at the limits of current surveys.

In all subsets considered, the luminosity of the $\mathrm{OH}$ masers is lower than that of $\mathrm{CH}_{3} \mathrm{OH}$ and $\mathrm{H}_{2} \mathrm{O}$ masers. The evolutionary phase during which $\mathrm{OH}$ maser emission occurs seems to follow those with $\mathrm{CH}_{3} \mathrm{OH} / \mathrm{H}_{2} \mathrm{O}$ emission, although an overlap of $\mathrm{OH}$ and $\mathrm{CH}_{3} \mathrm{OH}$ maser phases seems to exist.

The lowest percentage of association with radio continuum was found for objects with $\mathrm{CH}_{3} \mathrm{OH}$ masers alone, which seems to indicate that methanol masers (as $\mathrm{H}_{2} \mathrm{O}$ masers) may trace objects that have not yet developed an ionizing core. At least one third of the $\mathrm{H}_{2} \mathrm{O}$ masers in the sample have a velocity extent larger than $20 \mathrm{~km} \mathrm{~s}^{-1}$ suggesting their association with outflows or jets. For the majority of sources ( 90\%) the centroids of the $\mathrm{OH}$ and $\mathrm{CH}_{3} \mathrm{OH}$ maser velocity intervals coincide to within $5 \mathrm{~km} \mathrm{~s}^{-1}$. In contrast, for $\sim 40 \%$ of the $\mathrm{H}_{2} \mathrm{O}$ sources the difference in the velocity centroids of $\mathrm{H}_{2} \mathrm{O}$ and $\mathrm{CH}_{3} \mathrm{OH}$ maser emission are larger than $10 \mathrm{~km} \mathrm{~s}^{-1}$. Furthermore, the $\mathrm{H}_{2} \mathrm{O}$ velocity centroids are blue-shifted relative to those of $\mathrm{CH}_{3} \mathrm{OH}$ emission. These findings support previous suggestions that while $\mathrm{CH}_{3} \mathrm{OH}$ masers originate from the innermost parts of the environments of high-mass protostars, high velocity features are unique to $\mathrm{H}_{2} \mathrm{O}$ masers. These $\mathrm{H}_{2} \mathrm{O}$ masers likely emerge from high velocity outflows driven by the central object and can persist at a greater distance from exciting source than $\mathrm{CH}_{3} \mathrm{OH}$ masers.

The $\mathrm{OH}$ and $\mathrm{CH}_{3} \mathrm{OH}$ maser luminosities are well correlated with the IR luminosity while the $\mathrm{H}_{2} \mathrm{O}$ and IR luminosities are marginally correlated. These findings are fully consistent with pumping schemes of the three maser lines.

Acknowledgements. We thank T. K. Sridharan for providing maser data of his sample online. The work was supported by the KBN grant 2P03D01122.

\section{References}

Becker, R. H., White, R. L., Helfand, D. J., \& Zoonematkermani, S. 1994, ApJS, 91, 347

Beuther, H., Walsh, A., Schilke, P., et al. 2002, A\&A, 390, 289

Brand, J., \& Blitz, L. 1993, A\&A, 275, 67

Brand, J., Cesaroni, R., Comoretto, G., et al. 2003, A\&A, 407, 573

Bronfman, L., Nyman, L. A., \& May, J. 1996, A\&AS, 115, 81
Caswell, J. L. 1996, MNRAS, 279, 79

Caswell, J. L., Vaile, R. A., \& Forster, J. R. 1995, MNRAS, 277, 210

Charnley, S. B. 1997, ApJ, 481, 396

Charnley, S. B., Kress, M. E., Tielens, A. G. G. M., \& Millar, T. J. 1995, ApJ, 448, 232

Churchwell, E., Walmsley, C. M., \& Cesaroni, R. 1990, A\&AS, 83, 119

Codella, C., Felli, M., \& Natale, V. 1996, A\&A, 311, 971

Codella, C., Lorenzani, A., Gallego, A. T., Cesaroni, R., \& Moscadelli, L. 2004, A\&A, 417, 615

Codella, C., \& Moscadelli, L. 2000, A\&A, 362, 723

Codella, C., Palumbo, G. G. C., Pareschi, G., et al. 1995, MNRAS, 276, 57

Cragg, D. M., Sobolev, A. M., \& Godfrey, P. D. 2002, MNRAS, 331, 521

Elitzur, M., Hollenbach, D. J., \& McKee, C. F. 1989, ApJ, 346, 983

Ellingsen, S. P., von Bibra, M. L., McCulloch, P. M., et al. 1996, MNRAS, 280, 378

Forster, J. R., \& Caswell, J. L. 1989, A\&A, 213, 339

Hofner, P., \& Churchwell, E. 1996, A\&AS, 120, 283

Kolpak, M. A., Jackson, J. M., Bania, T. M., Clemens, D. P., \& Dickey, J. M. 2003, ApJ, 582, 756

Kurtz, S., Cesaroni, R., Churchwell, E., Hofner, P., \& Walmsley, C. M. 2000, in Protostars and Planets IV, ed. V. Mannings, A. P. Boss, \& S. S. Russell (Univ. of Arizona Press), 299

Menten, K. M. 1991, ApJ, 380, L75

Menten, K. M. 1996, Molecules in Astrophysics: Probes \& Processes, IAU Symp., 178, 163

Menten, K. M., Reid, M. J., Pratap, P., Moran, J. M., \& Wilson, T. L. 1992, ApJ, 401, L39

Minier, V., Ellingsen, S. P., Norris, R. P., \& Booth, R. S. 2003, A\&A, 403, 1095

Palla, F., Brand, J., Cesaroni, R., Comoretto, G., \& Felli, M. 1991, A\&A, 246, 249

Palla, F., Cesaroni, R., Brand, J., et al. 1993, A\&A, 280, 599

Reid, M. J., \& Moran, J. M. 1988, in Galactic and extra-galactic radio astronomy, ed. G. L. Verschuur, \& K. I. Kellermann (Berlin: Springer)

Sewilo, M., Watson, C., Araya, E., et al. 2004, ApJS, 154, 553

Schutte, A. J., van der Walt, D. J., Gaylard, M. J., \& MacLeod, G. C. 1993, MNRAS, 261, 783

Slysh, V. I., Val'tts, I. E., Kalenskii, S. V., et al. 1999, A\&AS, 134, 115

Sridharan, T. K., Beuther, H., Schilke, P., Menten, K. M., \& Wyrowski, F. 2002, ApJ, 566, 931

Szymczak, M., \& Gérard, E. 2004, A\&A, 414, 235

Szymczak, M., Kus, A. J., Hrynek, G., Kepa, A., \& Pazderski, E. 2002, A\&A, 392, 277

Szymczak, M., Hrynek, G., \& Kus, A. J. 2000, A\&AS, 143, 269

Valdettaro, R., Palla, F., Brand, J., et al. 2001, A\&AS, 368, 845

van der Walt, D. J., Gaylard, M. J., \& MacLeod, G. C. 1995, A\&AS, 110,81

van der Walt, D. J., Retief, S. J. P., Gaylard, M. J., \& MacLeod, G. C. 1996, MNRAS, 282, 1085

Walsh, A. J., Hyland, A. R., Robinson, G., \& Burton, M. G. 1997, MNRAS, 291, 261

Walsh, A. J., Burton, M. G., Hyland, A. R., \& Robinson, G. 1998, MNRAS, 301, 640

Walsh, A. J., Bertoldi, F., Burton, M. G., \& Nikola, T. 2001, MNRAS, 326, 36

Wood, D. O. S., \& Churchwell, E. 1989, ApJ, 340, 265 (WC89)

Wouterlout, J. G. A., \& Walmsley, C. M. 1986, A\&A, 168, 237

Wyrowski, F., Schilke, P., Walmsley, C. M., \& Menten, K. M. 1999, ApJ, 514, L43 